\title{
The edit distance function and symmetrization
}

\author{
Ryan Martin* \\ Department of Mathematics \\ Iowa State University \\ Ames, Iowa, U.S.A. \\ rymartin@iastate.edu
}

Submitted: April 17, 2012; Accepted: Aug 20, 2013; Published: Aug 30, 2013

Mathematics Subject Classifications: 05C35, 05C80

\begin{abstract}
The edit distance between two graphs on the same labeled vertex set is the size of the symmetric difference of the edge sets. The distance between a graph, $G$, and a hereditary property, $\mathcal{H}$, is the minimum of the distance between $G$ and each $G^{\prime} \in \mathcal{H}$ with the same number of vertices. The edit distance function of $\mathcal{H}$ is a function of $p \in[0,1]$ and is the limit of the maximum normalized distance between a graph of density $p$ and $\mathcal{H}$.

This paper utilizes a method due to Sidorenko [Combinatorica 13(1), pp. 109120], called "symmetrization", for computing the edit distance function of various hereditary properties. For any graph $H, \operatorname{Forb}(H)$ denotes the property of not having an induced copy of $H$. This paper gives some results regarding estimation of the function for an arbitrary hereditary property. This paper also gives the edit distance function for $\operatorname{Forb}(H)$, where $H$ is a cycle on 9 or fewer vertices.
\end{abstract}

Keywords: edit distance, hereditary properties, symmetrization, cycles, colored regularity graphs, quadratic programming

\section{Introduction}

The study of the edit distance in graphs originated independently by Axenovich, Kézdy and the author [6], Alon and Stav [2] and, in a different formulation, by Richer [17]. Since then, there has been a great deal of study on the edit distance itself and on the so-called edit distance function.

\footnotetext{
*This author's research partially supported by NSF grant DMS-0901008 and by an Iowa State University Faculty Professional Development grant.
} 


\subsection{The edit distance function}

The edit distance between graphs $G$ and $G^{\prime}$ on the same labeled vertex set is $\left|E(G) \triangle E\left(G^{\prime}\right)\right|$ and is denoted $\operatorname{dist}\left(G, G^{\prime}\right)$. The distance between a graph $G$ and a property $\mathcal{H}$ is

$$
\operatorname{dist}(G, \mathcal{H}):=\min \left\{\operatorname{dist}\left(G, G^{\prime}\right): V(G)=V\left(G^{\prime}\right), G^{\prime} \in \mathcal{H}\right\} .
$$

The edit distance function of a property $\mathcal{H}$, denoted $e d_{\mathcal{H}}(p)$, measures the maximum distance of a density $p$ graph from $\mathcal{H}$. Formally,

$$
e d_{\mathcal{H}}(p)=\lim _{n \rightarrow \infty} \max \left\{\operatorname{dist}(G, \mathcal{H}):|V(G)|=n,|E(G)|=\left\lfloor p\left(\begin{array}{l}
n \\
2
\end{array}\right)\right\rfloor\right\} /\left(\begin{array}{c}
n \\
2
\end{array}\right)
$$

if this limit exists.

A hereditary property is a family of graphs that is closed under the taking of induced subgraphs. It is natural to study the edit distance of graphs from hereditary properties because if $H$ is an induced subgraph of $G$ and $H^{\prime}$ is an induced subgraph of $G^{\prime}$, then $\operatorname{dist}\left(H, H^{\prime}\right) \leqslant \operatorname{dist}\left(G, G^{\prime}\right)$.

A hereditary property $\mathcal{H}$ is trivial if there is an $n_{0}$ such that $\mathcal{H}$ has no $n_{0}$-vertex graph (hence, no $n$-vertex graph for $n \geqslant n_{0}$ ). Otherwise, it is nontrivial. If $\mathcal{H}$ is a nontrivial hereditary property, then it has an $n$-vertex graph for all natural numbers $n$. Throughout this paper, all graph properties will be nontrivial hereditary properties.

In [8], a result of Alon and Stav [2] is generalized to show that the limit in (1) does indeed exist for nontrivial hereditary properties and, furthermore, that is the limit of the expectation of the edit distance function for random graphs with the appropriate edgeprobability. We use $G(n, p)$ to denote the Erdös-Rényi random graph on $n$ vertices with edge probability $p$.

$$
e d_{\mathcal{H}}(p)=\lim _{n \rightarrow \infty} \mathbb{E}[\operatorname{dist}(G(n, p), \mathcal{H})] /\left(\begin{array}{c}
n \\
2
\end{array}\right) .
$$

It is explicitly shown in [8] that, for any nontrivial hereditary property $\mathcal{H}$, the function $e d_{\mathcal{H}}(p)$ is continuous and concave down. Hence, it achieves its maximum. We let $d_{\mathcal{H}}^{*} \stackrel{\text { def }}{=}$ $\max _{p \in[0,1]} e d_{\mathcal{H}}(p)$ and $p_{\mathcal{H}}^{*}$ to be the set of all values of $p$ for which $e d_{\mathcal{H}}(p)$ achieves the maximum. In most cases, $p_{\mathcal{H}}^{*}$ is a single value and in that case, we will just refer to $p_{\mathcal{H}}^{*}$ as a real number rather than as a set.

A principal hereditary property is the set of all graphs with no induced copy of some fixed graph. The principal hereditary property $\operatorname{Forb}(H)$ denotes the set of graphs with no induced copy of $H$. In fact, every hereditary property is an intersection of principal hereditary properties. We denote $\mathcal{F}(\mathcal{H})$ to be the forbidden graphs for $\mathcal{H}$; that is, the minimal (with respect to vertex-deletion) set of graphs $H$ for which

$$
\mathcal{H}=\bigcap_{H \in \mathcal{F}(\mathcal{H})} \operatorname{Forb}(H)
$$

If $\mathcal{H}=\bigcap_{H \in \mathcal{F}(\mathcal{H})} \operatorname{Forb}(H)$, then we denote $\overline{\mathcal{H}}$ to be the hereditary property that is $\overline{\mathcal{H}}=$ $\bigcap_{H \in \mathcal{F}(\mathcal{H})} \operatorname{Forb}(\bar{H})$. I.e., $H \in \mathcal{F}(\mathcal{H})$ if and only if $\bar{H} \in \mathcal{F}(\overline{\mathcal{H}})$. Note that $\overline{\mathcal{H}}$ does not denote the complement of $\mathcal{H}$ as a set. 
For background on the edit distance function, applications thereof and theoretical background, we direct the reader to Balogh and the author [8], Alon and Stav [2, 3, 4, 5], Axenovich, Kézdy and the author [6], and Axenovich and the author [7]. The theoretical background upon which this is based can be traced to papers by Prömel and Steger [14, 15, 16], Bollobás and Thomason [9, 10] and Alekseev [1], among others.

\subsection{Main results}

Theorem 1 utilizes known techniques and observations to obtain bounds for hereditary properties of graphs with no clique of a given size. Theorem 2 uses the same ideas, in addition to the technique of known as symmetrization. Discussed in depth in Section 5.2, symmetrization is the main idea of this paper.

Using the fact that $e d_{\mathcal{H}}(p)=e d_{\overline{\mathcal{H}}}(1-p)$ (observed in Theorem $10(\mathrm{v})$ ) we see that Theorem 1 can be applied to hereditary properties that forbid an independent set as well as hereditary properties that forbid a clique.

Theorem 1. Let $\mathcal{H}$ be a nontrivial hereditary property such that $\mathcal{F}(\mathcal{H})$ contains a complete graph and let $h$ be the minimum positive integer such that $\mathcal{H} \subseteq \operatorname{Forb}\left(K_{h}\right)$. Let $\chi$ be the chromatic number of $\mathcal{H}$ and $m$ be the smallest positive integer such that $\mathcal{F}(\mathcal{H})$ contains a complete multipartite graph with $m$ parts. Clearly, $\chi \leqslant m \leqslant h$.

$$
\min \left\{\frac{p}{\chi-1}, \frac{1-p}{\chi-1}+\frac{2 p-1}{m-1}\right\} \leqslant e d_{\mathcal{H}}(p) \leqslant \min \left\{\frac{p}{\chi-1}, 1-p+\frac{2 p-1}{m-1}\right\} .
$$

In particular,

$$
e d_{\operatorname{Forb}\left(K_{h}\right)}(p)=\frac{p}{\chi-1}
$$

In Theorem 2, equation (2) is a trivial result, equation (3) was proven by Marchant and Thomason [12]. Some related results for $C_{4}$ were obtained by Alon and Stav [3]. Thomason [19] reports that Marchant has proven equation (4) and (6). We note that the problem considered in [12] is not edit distance but can be shown to be equivalent. Equations (5), (7) and (9) are entirely new and (4) and (6) are proved using symmetrization.

Theorem 2. Let $C_{h}$ denote the cycle on $h$ vertices.

$$
\begin{aligned}
e d_{\mathrm{Forb}\left(C_{3}\right)}(p) & =\frac{p}{2} \\
e d_{\mathrm{Forb}\left(C_{4}\right)}(p) & =p(1-p) \\
e d_{\mathrm{Forb}\left(C_{5}\right)}(p) & =\min \left\{\frac{p}{2}, \frac{1-p}{2}\right\} \\
e d_{\mathrm{Forb}\left(C_{6}\right)}(p) & =\min \left\{p(1-p), \frac{1-p}{2}\right\} \\
e d_{\mathrm{Forb}\left(C_{7}\right)}(p) & =\min \left\{\frac{p}{2}, \frac{p(1-p)}{1+p}, \frac{1-p}{3}\right\}
\end{aligned}
$$




$$
\begin{aligned}
e d_{\mathrm{Forb}\left(C_{8}\right)}(p) & =\min \left\{\frac{p(1-p)}{1+p}, \frac{1-p}{3}\right\} \\
e d_{\mathrm{Forb}\left(C_{9}\right)}(p) & =\min \left\{\frac{p}{2}, \frac{1-p}{4}\right\} \\
e d_{\mathrm{Forb}\left(C_{10}\right)}(p) & =\min \left\{\frac{p(1-p)}{1+2 p}, \frac{1-p}{4}\right\}, \quad \text { for } p \in[1 / 7,1] .
\end{aligned}
$$

Corollary 3. Let $C_{h}$ denote the cycle on $h$ vertices. Then,

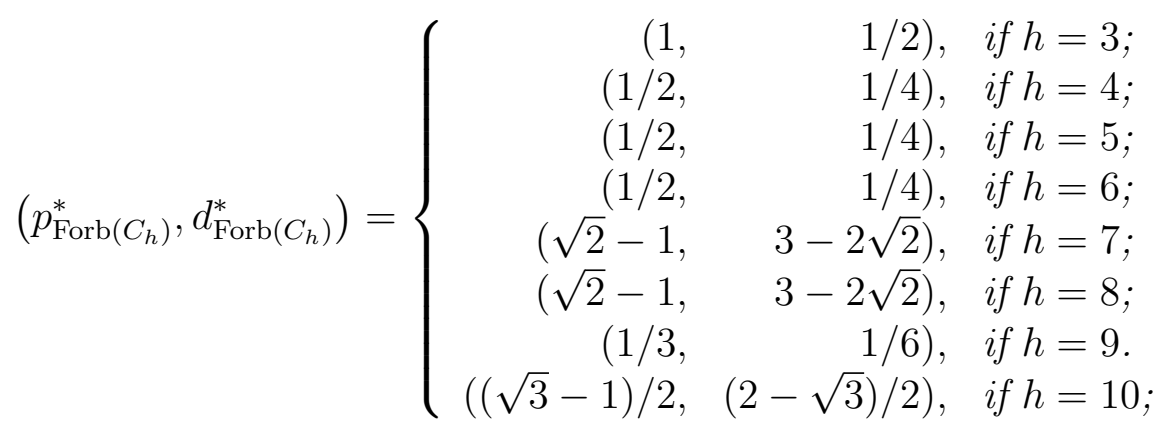

The rest of the paper is organized as follows: Section 2 gives some of the general definitions for the edit distance function, such as colored regularity graphs. Section 3 gives some theorems with which the edit distance function can be estimated. Section 4 contains the proof of Theorem 1. Section 5.1 defines and categorizes so-called $p$-core colored regularity graphs introduced by Marchant and Thomason [12]. Section 5.2 discusses the symmetrization method in general. Section 6 proves Theorem 2 regarding cycles. Section 7 gives some concluding remarks, a conjecture and acknowledgements.

\section{Development of the proofs}

\subsection{Notation}

All graphs are simple. If $S$ and $T$ are sets, then $S \cup T$ denotes the disjoint union of $S$ and $T$. If $G_{1}$ and $G_{2}$ are graphs, then $G_{1} \cup G_{2}$ denotes the disjoint union of the graphs and $G_{1} \vee G_{2}$ denotes the join. If $v$ and $w$ are adjacent vertices in a graph, we denote the edge between them to be $v w$.

\subsection{Colored regularity graphs}

A colored regularity graph $(C R G), K$, is a simple complete graph, together with a partition of the vertices into white and black $V(K)=\mathrm{VW}(K) \cup \mathrm{VB}(K)$ and a partition of the edges into white, gray and black $E(K)=\mathrm{EW}(K) \cup \operatorname{EG}(K) \cup \operatorname{EB}(K)$. We say that a graph $H$ embeds in $K$, (writing $H \mapsto K$ ) if there is a function $\varphi: V(H) \rightarrow V(K)$ so that if $h_{1} h_{2} \in E(H)$, then either $\varphi\left(h_{1}\right)=\varphi\left(h_{2}\right) \in \mathrm{VB}(K)$ or $\varphi\left(h_{1}\right) \varphi\left(h_{2}\right) \in \operatorname{EB}(K) \cup \operatorname{EG}(K)$ and if $h_{1} h_{2} \notin E(H)$, then either $\varphi\left(h_{1}\right)=\varphi\left(h_{2}\right) \in \mathrm{VW}(K)$ or $\varphi\left(h_{1}\right) \varphi\left(h_{2}\right) \in \mathrm{EW}(K) \cup \mathrm{EG}(K)$. 
For a hereditary property of graphs, we denote $\mathcal{K}(\mathcal{H})$ to be the subset of CRGs such that no forbidden graph maps into $K$. That is, $\mathcal{K}(\mathcal{H})=\{K: H \not \neg K, \forall H \in \mathcal{F}(\mathcal{H})\}$.

In a CRG, $K$, vertex $v$ is twin to vertex $w$ if their neighborhoods are the same. That is, they are twins if (a) $v$ and $w$ and $v w$ all have the same color and (b) whenever $x \in V(K)-\{v, w\}$, the edges $v x$ and $w x$ have the same color.

We say that a CRG, $K^{\prime}$ is formed by the partition of a vertex $v$ if $V\left(K^{\prime}\right)=V(K) \cup\left\{v^{\prime}\right\}$ where, for every $x \in V(K)$, the edge $v^{\prime} x$ has the same color in $K^{\prime}$ as $v x$ has in $K$. All other edges in $K^{\prime}$ inherit the same color as in $K$. We say that $K^{\prime \prime}$ is formed by the fusion of twin vertices $v$ and $v^{\prime}$ by letting $V\left(K^{\prime}\right)=V(K)-\left(\left\{v, v^{\prime}\right\}\right) \cup\left\{v^{\prime \prime}\right\}$ where, for every $x \in V(K)$, the edge $v^{\prime \prime} x$ has the same color as both $v x$ and $v^{\prime} x$.

Two CRGs, $K$ and $K^{\prime}$ are said to be equivalent if $K^{\prime}$ can be constructed from $K$ by the partition of vertices or fusion of twin vertices. A CRG is reduced if it has no pair of twin vertices.

A CRG $K^{\prime}$ is said to be $a s u b-C R G$ of $K$ if $K^{\prime}$ can be obtained by deleting vertices of $K$.

\subsection{The $f$ and $g$ functions}

For every hereditary property, $\mathcal{H}$, the function $e d_{\mathcal{H}}(p)$ in $(1)$, measures not only the maximum normalized edit distance among density- $p$ graphs but also the expectation of the normalized distance from $G(n, p)$. That is, Alon and Stav [2] prove that

$$
e d_{\mathcal{H}}(p)=\lim _{n \rightarrow \infty} \mathbb{E}[\operatorname{dist}(G(n, p), \mathcal{H})] /\left(\begin{array}{l}
n \\
2
\end{array}\right) .
$$

The normalized distance of $G(n, p)$ from a hereditary property is well-defined because the distance from $G(n, p)$ to $\mathcal{H}$ is concentrated around its mean.

For every CRG, $K$, we associate two functions of $p \in[0,1]$. The function $f$ is linear in $p$ and $g$ is found by the solution of a quadratic program. Let $K$ have a total of $k$ vertices $\left\{v_{1}, \ldots, v_{k}\right\}$, and let $\mathbf{M}_{K}(p)$ be a matrix such that the entries are:

$$
\left[\mathbf{M}_{K}(p)\right]_{i j}= \begin{cases}p, & \text { if } v_{i} v_{j} \in \mathrm{EW}(K) \text { or } v_{i}=v_{j} \in \mathrm{VW}(K) \\ 1-p, & \text { if } v_{i} v_{j} \in \operatorname{EB}(K) \text { or } v_{i}=v_{j} \in \mathrm{VB}(K) \\ 0, & \text { if } v_{i} v_{j} \in \operatorname{EG}(K)\end{cases}
$$

Then, we can express the $f$ and $g$ functions over the domain $p \in[0,1]$ as follows, with $\mathrm{VW}=\mathrm{VW}(K), \mathrm{VB}=\mathrm{VB}(K), \mathrm{EW}=\mathrm{EW}(K)$ and $\mathrm{EB}=\mathrm{EB}(K)$ :

$$
\begin{aligned}
f_{K}(p)= & \frac{1}{k^{2}}[p(|\mathrm{VW}|+2|\mathrm{EW}|)+(1-p)(|\mathrm{VB}|+2|\mathrm{~EB}|)] \\
g_{K}(p)= & \left\{\begin{aligned}
\min \quad \mathbf{x}^{T} \mathbf{M}_{K}(p) \mathbf{x} \\
\text { s.t. } \quad \mathbf{x}^{T} \mathbf{1}=1 \\
\mathbf{x} \geqslant \mathbf{0}
\end{aligned}\right.
\end{aligned}
$$

If we denote $\mathbf{1}$ to be the vector of all ones, then $f_{K}(p)=\left(\frac{1}{k} \mathbf{1}\right)^{T} \mathbf{M}_{K}(p)\left(\frac{1}{k} \mathbf{1}\right)$. So, $f_{K}(p) \geqslant$ $g_{K}(p)$. 
Fact 4. The function $g$ is invariant under equivalence classes of CRGs. That is, if $K$ and $K^{\prime}$ are equivalent $C R G$ s, then $g_{K}(p)=g_{K^{\prime}}(p)$ for all $p \in[0,1]$.

We can use both the $f$ and $g$ functions of CRGs to compute the edit distance function. Theorem 5 ([8]). For any nontrivial hereditary property $\mathcal{H}$,

$$
e d_{\mathcal{H}}(p)=\inf _{K \in \mathcal{K}(\mathcal{H})} g_{K}(p)=\inf _{K \in \mathcal{K}(\mathcal{H})} f_{K}(p) .
$$

Remark 6. Marchant and Thomason [12] prove that, in fact,

$$
e d_{\mathcal{H}}(p)=\min _{K \in \mathcal{K}(\mathcal{H})} g_{K}(p) .
$$

That is, that for every $p \in[0,1]$, there is a $C R G, K \in \mathcal{K}(\mathcal{H})$, such that ed $d_{\mathcal{H}}(p)=g_{K}(p)$.

A sub-CRG, $K^{\prime}$, of a CRG, $K$, is a component if, for all $v \in V\left(K^{\prime}\right)$ and all $w \in$ $V(K)-V\left(K^{\prime}\right)$, the edge $v w$ is gray. Theorem 7 allows the computation of $g_{K}$ from the $g$ functions of its components.

Theorem 7. Let $K$ be a $C R G$ with components $K^{(1)}, \ldots, K^{(\ell)}$. Then

$$
\left(g_{K}(p)\right)^{-1}=\sum_{i=1}^{\ell}\left(g_{K^{(i)}}(p)\right)^{-1} .
$$

Proof. The matrix $\mathbf{M}_{K}(p)$ is a block-diagonal matrix after permuting the vertices. Let $M_{K^{(i)}}(p)$ be the submatrix corresponding to the vertices of $K_{i}$. Let $g_{i}=g_{K^{(i)}}(p)$ for $i=1, \ldots, \ell$ and $g=g_{K}(p)$.

In the computation of $g$, we observe that the optimal choice of entries that correspond to the vertices of $K_{i}$ are not dependent on the choice of the assignments to vertices outside of $K_{i}$ but is only dependent on the total sum of the entries assigned to the vertices $K_{i}$. To wit,

$$
\begin{aligned}
g & =\min \left\{\mathbf{x}^{T} M_{K}(p) \mathbf{x}: \mathbf{x}^{T} \mathbf{1}=1, \mathbf{x} \geqslant \mathbf{0}\right\} \\
& =\min \left\{\sum_{i=1}^{\ell} \min \left\{\mathbf{x}_{i}^{T} M_{K^{(i)}}(p) \mathbf{x}_{i}: \mathbf{x}_{i}^{T} \mathbf{1}=\alpha_{i}, \mathbf{x}_{i} \geqslant \mathbf{0}\right\}: \alpha_{1}+\cdots+\alpha_{\ell}=1, \alpha_{i} \geqslant 0 \forall i\right\} .
\end{aligned}
$$

For each of the entries of the sum,

$$
\min \left\{\mathbf{x}_{i}^{T} M_{K^{(i)}}(p) \mathbf{x}_{i}: \mathbf{x}_{i}^{T} \mathbf{1}=\alpha_{i}, \mathbf{x}_{i} \geqslant \mathbf{0}\right\}=\alpha_{i}^{2} g_{i} .
$$

Consequently, $g$ is computed via the following optimization problem:

$$
g=\left\{\begin{array}{rrr}
\min & \alpha_{1}^{2} g_{1}+\cdots+\alpha_{\ell}^{2} g_{\ell} & \\
\text { s.t. } & \alpha_{1}+\cdots+\alpha_{\ell} & =1 \\
& \alpha_{1}, \ldots, \alpha_{\ell} & \geqslant 0
\end{array}\right.
$$

Using the method of Lagrange multipliers, we see that the solution is $\alpha_{i}=\lambda / g_{i}$ for $i=$ $1, \ldots, \ell$ and $\lambda^{-1}=\sum_{i=1}^{\ell} g_{i}^{-1}$. Substituting these values gives the theorem statement. 
Theorem 7 can be applied directly to CRGs that have only gray edges. Since the $g$ function for a white vertex is $p$ and the $g$ function for a black vertex is $1-p$, we have Corollary 8:

Corollary 8. If $K$ is a CRG all of whose edges are gray, then

$$
g_{K}(p)=\left(\frac{|\mathrm{VW}(K)|}{p}+\frac{|\mathrm{VB}(K)|}{1-p}\right)^{-1} .
$$

Proposition 9 gives the edit distance function for some special CRGs that have no gray edges.

Proposition 9. Let $K$ be a $C R G$ on $k$ vertices and no gray edges as follows:

- If all vertices are white and all edges are black, then $g_{K}(p)=\min \{p, 1-p+(2 p-$ $1) / k\}$.

- If all vertices are black and all edges are white, then $g_{K}(p)=\min \{p+(1-2 p) / k, 1-$ $p\}$.

\section{Estimation of the edit distance function}

Denote $K(r, s)$ to be the CRG with $r$ white vertices, $s$ black vertices and all gray edges. Let $\mathcal{H}$ be a hereditary property with $\mathcal{H}=\bigcap_{H \in \mathcal{F}(\mathcal{H})} \operatorname{Forb}(H)$. The notion of $(r, s)$-colorability is discussed by Alon and Stav [3] where they focus on hereditary properties that are complement-invariant.

The chromatic number of $\mathcal{H}$, denoted $\chi(\mathcal{H})$ or just $\chi$, where the context is clear, is $\min \{\chi(H): H \in \mathcal{F}(\mathcal{H})\}$. The complementary chromatic number ${ }^{1}$ of $\mathcal{H}$, denoted $\bar{\chi}(\mathcal{H})$ or $\bar{\chi}$, is $\min \{\chi(\bar{H}): H \in \mathcal{F}(\mathcal{H})\}$. The binary chromatic number is

$$
\max \{k+1: \exists r, s, r+s=k, H \not p K(r, s), \forall H \in \mathcal{F}(\mathcal{H})\} .
$$

The clique spectrum of $\mathcal{H}$ is the set

$$
\Gamma(\mathcal{H}) \stackrel{\text { def }}{=}\{(r, s): H \not \rightarrow K(r, s), \forall H \in \mathcal{F}(\mathcal{H})\} .
$$

The clique spectrum has a number of useful properties. For example, it is monotone in the sense that if $(r, s) \in \Gamma(\mathcal{H})$ and $0 \leqslant r^{\prime} \leqslant r$ and $0 \leqslant s^{\prime} \leqslant s$, then $\left(r^{\prime}, s^{\prime}\right) \in \Gamma(\mathcal{H})$. As a result, the clique spectrum of a hereditary property can be expressed as a Young tableau. An extreme point of the clique spectrum $\Gamma$ is a pair $(r, s) \in \Gamma$ for which both $(r+1, s) \notin \Gamma$ and $(r, s+1) \notin \Gamma$. Let $\Gamma^{*}$ denote the extreme points of clique spectrum $\Gamma$. Figure 3 shows the clique spectrum of the cycle $C_{9}$ expressed as a Young tableau, with the extreme points of the clique spectrum marked.

\footnotetext{
${ }^{1}$ Unfortunately, the term "cochromatic number" is taken. It should be noted that the cochromatic number, although its definition resembles that of $\chi_{B}$, is not the same parameter.
} 


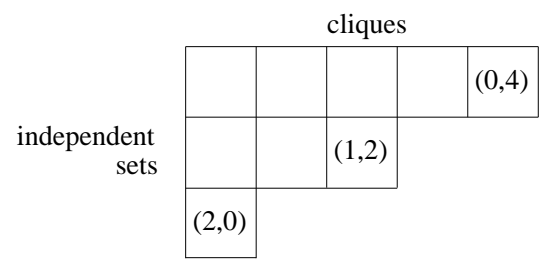

Figure 1: The clique spectrum of $C_{9}$ expressed as a Young tableau. The extreme points of the clique spectrum are labeled.

\subsection{Approximating $\boldsymbol{e} \boldsymbol{d}_{\mathcal{H}}(p)$ by $\gamma_{\mathcal{H}}(p)$}

Corollary 8 gives that $g_{K(r, s)}(p)=\frac{p(1-p)}{r(1-p)+s p}$, which follows directly from Theorem 7 . Define the function $\gamma_{\mathcal{H}}(p)$ as follows:

$$
\gamma_{\mathcal{H}}(p) \stackrel{\text { def }}{=} \min \left\{g_{K(r, s)}(p):(r, s) \in \Gamma(\mathcal{H})\right\}=\min \left\{\frac{p(1-p)}{r(1-p)+s p}:(r, s) \in \Gamma(\mathcal{H})\right\} .
$$

Clearly, $e d_{\mathcal{H}}(p) \leqslant \gamma_{\mathcal{H}}(p)$. Moreover, $\gamma_{\mathcal{H}}(p)=\min \left\{g_{K(r, s)}(p):(r, s) \in \Gamma^{*}(\mathcal{H})\right\}$; i.e, only $(r, s)$ that are extreme points of the clique spectrum need to be used to compute $\gamma$. The value of the function $\gamma_{\mathcal{H}}(p)$ is that it is computable for any hereditary property.

\subsection{Basic observations on $\operatorname{ed}_{\mathcal{H}}(p)$}

The following is a summary of basic facts about the edit distance function. Item (iii) comes from Alon and Stav [2]. Item (iv) comes from [8]. The remaining items are trivial.

Theorem 10. Let $\mathcal{H}$ be a nontrivial hereditary property with chromatic number $\chi$, complementary chromatic number $\bar{\chi}$, binary chromatic number $\chi_{B}$ and edit distance function $e d_{\mathcal{H}}(p)$.

i. If $\chi>1$, then $\operatorname{ed} d_{\mathcal{H}}(p) \leqslant p /(\chi-1)$.

ii. If $\bar{\chi}>1$, then $e d_{\mathcal{H}}(p) \leqslant(1-p) /(\bar{\chi}-1)$.

iii. $\operatorname{ed}_{\mathcal{H}}(1 / 2)=1 /\left(2\left(\chi_{B}-1\right)\right)=\gamma_{\mathcal{H}}(1 / 2)$.

iv. $e d_{\mathcal{H}}(p)$ is continuous and concave down.

v. $e d_{\mathcal{H}}(p)=e d_{\overline{\mathcal{H}}}(1-p)$.

Proof. Item (i) follows from the fact that $(\chi-1,0)$ is in the clique spectrum $\Gamma(\mathcal{H})$. That is, we can partition $V(G)$ into $\chi-1$ nearly-equally-sized pieces and delete the edges which have both endpoints in the same piece. The resulting graph is in $\mathcal{H}$. Item (ii) follows similarly. Item (iii) comes from Alon and Stav [2]. Item (iv) comes from [8].

Item (v) comes from the fact that if $H$ is a graph and $K$ is a CRG, then $H \not \neg K$ if and only if $\bar{H} \not \neg \bar{K}$, where $\bar{K}$ is the natural complement of the CRG $K$ : $\operatorname{VW}(\bar{K})=\mathrm{VB}(K)$, $\mathrm{VB}(\bar{K})=\mathrm{VW}(K), \mathrm{EW}(\bar{K})=\mathrm{EB}(K), \mathrm{EG}(\bar{K})=\mathrm{EG}(K)$ and $\operatorname{VB}(\bar{K})=\mathrm{VW}(K)$. 
There are a number of immediate corollaries of Theorem 10 that help estimate the edit distance functions. Some of the most useful are summarized in Corollary 11 and we leave the proof of them to the reader.

Corollary 11. Let $\mathcal{H}$ be a nontrivial hereditary property with binary chromatic number $\chi_{B}$. Let $(r, s)$ be extreme points in the clique spectrum of $\mathcal{H}$ such that $r+s=\chi_{B}$.

i. If $\chi=\chi_{B}$, then $e d_{\mathcal{H}}(p)=p /(\chi-1)$ for all $p \in[0,1 / 2]$.

ii. If $\chi_{B}=\bar{\chi}$, then $e d_{\mathcal{H}}(p)=(1-p) /\left(\chi_{B}-1\right)$ for all $p \in[1 / 2,1]$.

iii. If $r \geqslant s$, then $p_{\mathcal{H}}^{*} \geqslant 1 / 2$.

iv. If $r \leqslant s$, then $p_{\mathcal{H}}^{*} \leqslant 1 / 2$.

$v$. For any $(r, s)$ in the clique spectrum, $d_{\mathcal{H}}^{*} \leqslant(\sqrt{r}+\sqrt{s})^{-2}$.

Proof. Theorem 10(i) gives that $e d_{\mathcal{H}}(p) \leqslant p /(\chi-1)$. Thus $e d_{\mathcal{H}}(0)=0$ and, since $\chi=\chi_{B}$, Theorem 10(iii) gives that $e d_{\mathcal{H}}(1 / 2)=1 /(2(\chi-1))$. Theorem 10(iv) allows us to conclude that $e d_{\mathcal{H}}(p) \geqslant p /(\chi-1)$, hence equality and item (i). Item (ii) follows from a symmetric argument.

As to the next two items, note that $g_{K(r, s)}(p)=\frac{p(1-p)}{r(1-p)+s p}$, which is a concave down function that achieves its maximum at the point $p=\frac{\sqrt{r}}{\sqrt{r}+\sqrt{s}}$. If $r \geqslant s$, then, for all $p \in[0,1 / 2)$, we have, via Theorem 10(iii),

$$
e d_{\mathcal{H}}(p) \leqslant g_{K(r, s)}(p)<g_{K(r, s)}(1 / 2)=1 /(2(r+s))=e d_{\mathcal{H}}(1 / 2) .
$$

Hence, $e d_{\mathcal{H}}(p)$ achieves its maximum for some $p \geqslant 1 / 2$ and item (iii) follows. Item (iii) follows from a symmetric argument.

For item (v), we just observe that $e d_{\mathcal{H}}(p) \leqslant g_{K(r, s)}(p)$, the maximum of which is the value $(\sqrt{r}+\sqrt{s})^{-2}$, achieved at $p=\frac{\sqrt{r}}{\sqrt{r}+\sqrt{s}}$.

\section{$4 \quad \mathcal{H} \subseteq \operatorname{Forb}\left(K_{h}\right)$}

In this section, we prove Theorem 1 , which bounds the edit distance function for hereditary properties that have no copy of a complete graph on $h$ vertices. Note that $\mathcal{H} \subseteq \operatorname{Forb}\left(K_{h}\right)$ if and only if $K_{h} \in \mathcal{F}(\mathcal{H})$.

Proof of Theorem 1. Since $\bar{\chi}(\mathcal{H})=1$ and $\mathcal{H}$ is not trivial, $\chi(\mathcal{H})>1$. If $K \in \mathcal{K}(\mathcal{H})$, then $K$ cannot have a black vertex, otherwise $K_{h} \mapsto K$. So, we may assume that $K \in \mathcal{K}(\mathcal{H})$ has all white vertices. In every set of $\chi$ white vertices, there must be a non-gray edge. By Turán's theorem, this means that $K$ has at least $\left(\begin{array}{l}k \\ 2\end{array}\right)-\frac{\chi-2}{\chi-1} \cdot \frac{k^{2}}{2}$ non-gray edges. Hence,

$$
e d_{\mathcal{H}}(p) \geqslant f_{K}(p) \geqslant \frac{1}{k^{2}}\left[p k+2 \min \{p, 1-p\}\left(\left(\begin{array}{l}
k \\
2
\end{array}\right)-\frac{\chi-2}{\chi-1} \cdot \frac{k^{2}}{2}\right)\right] \geqslant \frac{\min \{p, 1-p\}}{\chi-1} .
$$


In every set of $m$ white vertices, there must be a white edge. Again, by Turán's theorem, $e d_{\mathcal{H}}(p) \geqslant f_{K}(p) \geqslant \frac{p}{m-1}$. So, $e d_{\mathcal{H}}(p)$ is bounded below by both $p /(\chi-1)$ and the line segment connecting the points $\left(1 / 2, \frac{1}{2(\chi-1)}\right)$ and $\left(1, \frac{1}{m-1}\right)$. Hence,

$$
e d_{\mathcal{H}}(p) \geqslant \min \left\{\frac{p}{\chi-1}, \frac{1-p}{\chi-1}+\frac{2 p-1}{m-1}\right\} \text {. }
$$

As to the upper bound, we give two CRGs into which no $H \in \mathcal{F}(\mathcal{H})$ can map. The first is $K^{(1)}=K(\chi-1,0)$, the CRG with $\chi-1$ white vertices and all edges gray. By Corollary $8, g_{K^{(1)}}(p)=p /(\chi-1)$.

The second CRG, $K^{(2)}$, is $m-1$ white vertices and all black edges. If there were some $H \in \mathcal{F}(\mathcal{H})$ such that $H \mapsto K^{(2)}$, then $H$ would be a complete $(m-1)$-partite graph, which is forbidden by our choice of $m$. By Proposition $9, g_{K^{(2)}}(p)=\min \{p, 1-p+(2 p-$ $1) /(m-1)\}$. So,

$$
e d_{\mathcal{H}}(p) \leqslant \min \left\{\frac{p}{\chi-1}, p, 1-p+\frac{2 p-1}{m-1}\right\} .
$$

The final statement comes from the observation that if $\mathcal{H}=\operatorname{Forb}\left(K_{h}\right)$, then $\chi=m=$ $h$.

By Theorem 10(v) we have the similar result for empty graphs: Let $\mathcal{H}$ be a nontrivial hereditary property such that $\mathcal{F}(\mathcal{H})$ contains an empty graph and let $h$ be the minimum positive integer such that $\mathcal{H} \subseteq \operatorname{Forb}\left(\overline{K_{h}}\right)$. Let $\bar{\chi}$ be the complementary chromatic number ${ }^{2}$ of $\mathcal{H}$ and $m$ be the smallest positive integer such that $\mathcal{F}(\mathcal{H})$ contains a $m$ disjoint cliques. Clearly, $\bar{\chi} \leqslant m \leqslant h$.

$$
\min \left\{\frac{p}{\bar{\chi}-1}+\frac{1-2 p}{m-1}, \frac{1-p}{\bar{\chi}-1}\right\} \leqslant e_{\mathcal{H}}(p) \leqslant \min \left\{p+\frac{1-2 p}{m-1}, \frac{1-p}{\bar{\chi}-1}\right\} .
$$

\section{Symmetrization}

An optimal solution to the quadratic program in (11) is, in some sense, regular, as we will show in equation (12) below. The idea, observed by Sidorenko [18] was first called "symmetrization" by Pikhurko [13].

The fundamental observation in symmetrization is that there is a balance that results from an optimal solution as we see in Proposition 12, which is slightly more general than we need. Recall that $\mathbf{0}$ and $\mathbf{1}$ are the all zeros and all ones vectors, respectively. Furthermore, $\mathbf{x} \geqslant \mathbf{0}$ means that the inequality holds entrywise.

Proposition 12. Let $M$ be a real symmetric square matrix with nonnegative entries. If $x^{*}$ is an optimal solution to the quadratic program

$$
g=\min \left\{\mathbf{x}^{T} \mathbf{M} \mathbf{x}: \mathbf{x}^{T} \mathbf{1}=1, \mathbf{x} \geqslant \mathbf{0}\right\}
$$

\footnotetext{
${ }^{2}$ The term $\bar{\chi}(\mathcal{H})$ is, the smallest number, $k$, such that no member of $\mathcal{F}(\mathcal{H})$ can be partitioned into $k$ cliques. In fact, $\bar{\chi}(\mathcal{H})=\chi(\overline{\mathcal{H}})$.
} 
such that $\mathbf{x}^{*}$ has no zero entries, then

$$
\mathrm{Mx}^{*}=g 1
$$

Moreover, if no optimal solution has a zero entry, then $\mathbf{x}^{*}$ is unique.

Proof. Suppose $\mathbf{M x}^{*}$ is not a constant vector. That is, the $i^{\text {th }}$ entry is larger than the $j^{\text {th }}$ entry. Define the vector $\mathbf{z}$ so that $\mathbf{z}_{i}=-1, \mathbf{z}_{j}=+1$ and every other entry of $\mathbf{z}$ is zero. Let $\epsilon>0$ be small; we will establish how small shortly.

For any $\epsilon,\left(\mathbf{x}^{*}+\epsilon \mathbf{z}\right)^{T} \mathbf{1}=1$. If $\epsilon$ is small enough, then $\mathbf{x}^{*}+\epsilon \mathbf{z} \geqslant 0$; moreover,

$$
\begin{aligned}
\left(\mathbf{x}^{*}+\epsilon \mathbf{z}\right)^{T} \mathbf{M}\left(\mathbf{x}^{*}+\epsilon \mathbf{z}\right) & =\left(\mathbf{x}^{*}\right)^{T} \mathbf{M} \mathbf{x}^{*}+\epsilon\left(\mathbf{x}^{*}\right)^{T} \mathbf{M z}+\epsilon \mathbf{z}^{T} \mathbf{M} \mathbf{x}^{*}+\epsilon^{2} \mathbf{z}^{T} \mathbf{M z} \\
& =\left(\mathbf{x}^{*}\right)^{T} \mathbf{M} \mathbf{x}^{*}+2 \epsilon \mathbf{z}^{T} \mathbf{M} \mathbf{x}^{*}+\epsilon^{2} \mathbf{z}^{T} \mathbf{M z}<\left(\mathbf{x}^{*}\right)^{T} \mathbf{M} \mathbf{x}^{*},
\end{aligned}
$$

contradicting $\mathbf{x}^{*}$ being optimal. Thus, $\mathbf{M x}^{*}=\alpha \mathbf{1}$ for some $\alpha$ and so

$$
g=\left(\mathbf{x}^{*}\right)^{T} \mathbf{M} \mathbf{x}^{*}=\alpha\left(\mathbf{x}^{*}\right)^{T} \mathbf{1}=\alpha .
$$

As to uniqueness, suppose there are no optimal solutions with zero entries. Let $\mathbf{x}_{1}^{*}$ and $\mathbf{x}_{2}^{*}$ be feasible solutions with nonzero entries and set $\epsilon>0$ so that $\mathbf{y}^{*}=\frac{1}{1-\epsilon}\left(\mathbf{x}_{1}^{*}-\epsilon \mathbf{x}_{2}^{*}\right)$ satisfies $\left(\mathbf{y}^{*}\right)^{T} \mathbf{1} \geqslant 0$ and has at least one zero entry. Since $\left(\mathbf{y}^{*}\right)^{T} \mathbf{1}=1$ and $\left(\mathbf{y}^{*}\right)^{T} \mathbf{M y}^{*}=g$, this is an optimal solution with a zero entry. This contradiction gives that the optimal solution must be unique.

If we apply Proposition 12 to the quadratic program in (11), we see that the optimal solution may have zero entries. This corresponds to deleting a vertex from $K$. So we may restrict ourselves only to CRGs for which any optimal solution has only positive entries.

In this case, let us see how Proposition 12 effects a specific CRG. There are three different types of degrees of a vertex in a CRG. Let $K$ be a CRG and $v \in V(K)$ and let $\mathbf{x}$ be an optimal solution to the quadratic program in (11) so that $\mathbf{x}(v)$ is the entry of $\mathbf{x}$ corresponding to vertex $v$. The gray degree of $v, \mathrm{~d}_{\mathrm{G}}(v)$ is the sum of the weights of the vertices that are adjacent to $v$ via a gray edge. The white degree of $v, \mathrm{~d}_{\mathrm{W}}(v)$ is the sum of the weights of the vertices that are adjacent to $v$ via a white edge plus $\mathbf{x}(v)$ if $v$ is a white vertex. The black degree of $v, \mathrm{~d}_{\mathrm{B}}(v)$ is the sum of the weights of the vertices that are adjacent to $v$ via a black edge plus $\mathbf{x}(v)$ if $v$ is a black vertex. Consequently, $\mathrm{d}_{\mathrm{W}}(v)+\mathrm{d}_{\mathrm{G}}(v)+\mathrm{d}_{\mathrm{B}}(v)=1$ for all $v \in V(K)$.

With the notation above, equation (12) gives that that either $\mathbf{x}^{*}$ has an entry equal to zero or, for every $v \in V(K)$,

$$
p \mathrm{~d}_{\mathrm{W}}(v)+(1-p) \mathrm{d}_{\mathrm{B}}(v)=g_{K}(p) .
$$

Therefore, we are interested only in CRGs for which deleting a vertex gives a CRG with a larger value of $g$. A CRG, $K$, is a $p$-core $C R G$, or simply a $p$-core, if $g_{K}(p)<g_{K^{\prime}}(p)$ for all nontrivial sub-CRGs $K^{\prime}$ of $K$. 


\subsection{The $p$-core CRGs}

Marchant and Thomason established that $p$-cores can be easily classified:

Theorem 13 (Marchant-Thomason, [12]). Let $K$ be a p-core CRG.

- If $p=1 / 2$, then $K$ has all of its edges gray.

- If $p<1 / 2$, then $\mathrm{EB}(K)=\emptyset$ and there are no white edges incident to white vertices.

- If $p>1 / 2$, then $\mathrm{EW}(K)=\emptyset$ and there are no black edges incident to black vertices.

By Theorem 13, if $K$ is a $p$-core CRG, then no edge has the same color as either of its endvertices, so we can simplify the definitions of $\mathrm{d}_{\mathrm{W}}$ and $\mathrm{d}_{\mathrm{B}}$ for $p$-cores as follows:

$$
\begin{aligned}
& \mathrm{d}_{\mathrm{W}}(v)= \begin{cases}\mathbf{x}(v), & \text { if } v \in \operatorname{VW}(K) ; \\
\sum_{v z \in \operatorname{EW}(K)} \mathbf{x}(z), & \text { if } v \in \operatorname{VB}(K) ;\end{cases} \\
& \mathrm{d}_{\mathrm{B}}(v)= \begin{cases}\sum_{v z \in \operatorname{EB}(K)} \mathbf{x}(z), & \text { if } v \in \operatorname{VW}(K) ; \\
\mathbf{x}(v), & \text { if } v \in \operatorname{VB}(K) .\end{cases}
\end{aligned}
$$

Of course, it is still the case that $\mathrm{d}_{\mathrm{G}}(v)=1-\mathrm{d}_{\mathrm{W}}(v)-\mathrm{d}_{\mathrm{B}}(v)$.

We can summarize the above in the following:

Theorem 14 (Marchant-Thomason, [12]). Let $K$ be a p-core CRG. There is a unique vector $\mathbf{x}$ that is an optimal solution to the quadratic program in (11). For all $v \in V(K)$, let the entry of $\mathbf{x}$ corresponding to $v$ be $\mathbf{x}(v)$ and let $\mathrm{d}_{\mathrm{W}}(v)$ and $\mathrm{d}_{\mathrm{B}}(v)$ be defined as above. For each $v \in V(K)$,

$$
g_{K}(p)=p \mathrm{~d}_{\mathrm{W}}(v)+(1-p) \mathrm{d}_{\mathrm{B}}(v) .
$$

\subsection{Computing edit distance functions using symmetrization}

Theorem 14, Theorem 13, Remark 6 and the definition of $p$-cores have all of the elements in order to express $\mathrm{d}_{\mathrm{G}}(v)=1-\mathrm{d}_{\mathrm{W}}(v)-\mathrm{d}_{\mathrm{B}}(v)$ for any vertex $v$ in a $p$-core CRG. It is often useful and intuitive to focus on the gray neighborhood of vertices.

Lemma 15. Let $p \in(0,1)$ and $K$ be a p-core $C R G$ with optimal weight function $\mathbf{x}$.

i. If $p \leqslant 1 / 2$, then, $\mathbf{x}(v)=g_{K}(p) / p$ for all $v \in \mathrm{VW}(K)$ and

$$
\mathrm{d}_{\mathrm{G}}(v)=\frac{p-g_{K}(p)}{p}+\frac{1-2 p}{p} \mathbf{x}(v), \quad \text { for all } v \in \mathrm{VB}(K) .
$$

ii. If $p \geqslant 1 / 2$, then $\mathbf{x}(v)=g_{K}(p) /(1-p)$ for all $v \in \mathrm{VB}(K)$ and

$$
\mathrm{d}_{\mathrm{G}}(v)=\frac{1-p-g_{K}(p)}{1-p}+\frac{2 p-1}{1-p} \mathbf{x}(v), \quad \text { for all } v \in \mathrm{VW}(K) .
$$


Proof. We will prove the case for $p \leqslant 1 / 2$. The case where $p \geqslant 1 / 2$ is symmetric. Let $v \in \mathrm{VW}(K)$. By Theorem 13, all vertices are incident to $v$ via a gray edge, and by Theorem 14, $g_{K}(p)=p \mathbf{x}(v)$. Now let $v \in \mathrm{VB}(K)$. By Theorem 13, $v$ has no black neighbors and

$$
g_{K}(p)=p\left(1-\mathbf{x}(v)-\mathrm{d}_{\mathrm{G}}(v)\right)+(1-p) \mathbf{x}(v) .
$$

Solving for $\mathrm{d}_{\mathrm{G}}(v)$ gives the result.

Lemma 16. Let $p \in(0,1)$ and $K$ be a p-core $C R G$ with optimal weight function $\mathbf{x}$.

i. If $p \leqslant 1 / 2$, then $\mathbf{x}(v) \leqslant g_{K}(p) /(1-p)$ for all $v \in \mathrm{VB}(K)$.

ii. If $p \geqslant 1 / 2$, then $\mathbf{x}(v) \leqslant g_{K}(p) / p$ for all $v \in \mathrm{VW}(K)$.

Proof. We use the fact that $\mathbf{x}(v)+\mathrm{d}_{\mathrm{G}}(v) \leqslant 1$. Applying Lemma 15 and solving for $\mathbf{x}(v)$ gives the result.

Remark 17. From this point forward in the paper, if $K$ is a CRG under consideration and $p$ is fixed, $\mathbf{x}(v)$ will denote the weight of $v \in V(K)$ under the optimal solution of the quadratic program in equation (11) that defines $g_{K}$.

\section{$6 \operatorname{Forb}\left(C_{h}\right), h \in\{3, \ldots, 9\}$}

Thomason [19] reports that Ed Marchant has found the edit distance function for $C_{5}$ and $C_{7}$. Here we find the function for all $C_{h}, h \in\{3, \ldots, 9\}$. The proofs in this section might be substantially similar to Marchant's.

In order to compute the edit distance function for cycles, we first make the observation that $C_{3}$ is a complete graph and so Theorem 1 gives Corollary 18.

\section{Corollary 18.}

$$
e d_{\mathrm{Forb}\left(C_{3}\right)}(p)=p / 2 \text {. }
$$

Furthermore, the only $p$-core for which this is achieved for $p \in(0,1)$ is $K(2,0)$.

For $C_{h}, h \geqslant 4$, we first take care of easy cases so that the only $p$-cores that need to be considered have all black vertices. We use Lemma 19 which establishes the upper bound and eliminates all cases except when $p \leqslant 1 / 2$ and all vertices are black.

Lemma 19. Let $h \geqslant 4$. For all $p \in(0,1)$,

$$
\gamma_{\text {Forb }\left(C_{h}\right)}(p)= \begin{cases}p(1-p), & \text { if } h=4 ; \\ \min \left\{\frac{p(1-p)}{1-p+(\lceil h / 3\rceil-1) p}, \frac{1-p}{\lceil h / 2\rceil-1}\right\}, & \text { if } h \geqslant 6 \text { is even; and } \\ \min \left\{\frac{p}{2}, \frac{p(1-p)}{1-p+(\lceil h / 3\rceil-1) p}, \frac{1-p}{\lceil h / 2\rceil-1}\right\}, & \text { if } h \text { is odd. }\end{cases}
$$

Furthermore, if there is a $p$-core $C R G, K \in \mathcal{K}\left(\operatorname{Forb}\left(C_{h}\right)\right)$ such that $g_{K}(p)<\gamma_{\operatorname{Forb}\left(C_{h}\right)}(p)$ for any $p \in(0,1)$, then $p<1 / 2$ and $K$ has all black vertices. 
Proof. Since $\chi\left(C_{h}\right)=\lceil h / 2\rceil$, the clique spectrum of $\operatorname{Forb}\left(C_{h}\right)$ contains $(0,0), \ldots$, $(0,\lceil h / 2\rceil-1)$. The vertex set of $C_{h}$ can be partitioned into 1 independent set and $\lceil h / 3\rceil$ cliques but not into $\lceil h / 3\rceil-1$ cliques. So, the clique spectrum of $\operatorname{Forb}\left(C_{h}\right)$ contains $(1,0), \ldots,(1,\lceil h / 3\rceil-1)$. Since the vertex set of $C_{h}$ can always be partitioned into 2 independent sets and a vertex, the clique spectrum of Forb $\left(C_{h}\right)$ can contain at most one more element - the pair $(2,0)$ in the case $h$ is odd.

Therefore, the extreme points of the clique spectrum of Forb $\left(C_{h}\right)$ are $(0,\lceil h / 2\rceil-1)$, $(1,\lceil h / 3\rceil-1)$ and, if $h$ is odd, $(2,0)$. This establishes the value of $\gamma_{\operatorname{Forb}\left(C_{h}\right)}(p)$ as outlined in the statement of the lemma.

If $h=4$, the classes of possible CRGs are restricted. If $K$ has at least 2 white vertices, they are connected via a gray or black edge and so $C_{4}$ would embed in $K$. If $K$ has a white and at least two black vertices, then the edges between the white and black vertices are both gray and the edge between the black vertices is either gray or white and so $C_{4}$ would embed in $K$. Thus, if $K$ has a white vertex, then it has at most one black vertex and this is $K(1,1)$, the CRG that defines $\gamma_{\operatorname{Forb}\left(C_{4}\right)}(p)=p(1-p)$. If $K$ has all white edges, then $g_{K}(p)=\min \{p+(1-2 p) / k, 1-p\}>p(1-p)$. So, $e d_{\operatorname{Forb}\left(C_{4}\right)}(p)=p(1-p)$.

Now, let $h \geqslant 5$. Since $\gamma_{\mathcal{H}}(1 / 2)=e d_{\mathcal{H}}(1 / 2)$ for all hereditary properties and $0=$ $\gamma_{\text {Forb }\left(C_{h}\right)}(1)$, convexity gives that $e d_{\mathcal{H}}(p)=\frac{1-p}{\lceil h / 2\rceil-1}$, for all $p \geqslant 1 / 2$.

Finally, let $p \in(0,1 / 2)$ and $K$ be a $p$-core CRG such that $C_{h} \not p h$. If $K$ has only white vertices and $h$ is even, then $K \approx K(1,0)$ and $g_{K}(p)=p>\gamma_{\mathcal{H}}(p)$. If $K$ has only white vertices and $h$ is odd, then there are at most 2 white vertices and $g_{K}(p) \geqslant p / 2$ with equality if and only if $K \approx K(2,0)$.

If $K$ has both white and black vertices, then it has at most 1 white vertex because $C_{h} \mapsto K(2,1)$. Furthermore, it can have at most $\lceil h / 3\rceil-1$ black vertices. To see this, denote the vertices of $C_{h}$ by $\{0,1, \ldots, h-1\}$ where $0 \sim 1 \sim \cdots \sim h-1 \sim 0$. Let $S$ consist of the members of $\{0, \ldots, h-2\}$ that are divisible by 3 . If $h-1$ is divisible by 3 , then add $h-2$ to $S$. The graph $C_{h}-S$ has $\lceil h / 3\rceil$ connected components, each of which are cliques of size 1 or 2 . Thus, regardless of whether the edges are white or gray, there are at most $\lceil h / 3\rceil-1$ black vertices in $K$ and $g_{K}(p) \geqslant \frac{p(1-p)}{1-p+(\lceil h / 3\rceil-1) p}$, with equality if and only if $K \approx K(1,\lceil h / 3\rceil-1)$.

Summarizing, if $p \in(0,1 / 2)$ and $g_{K}(p)=e d_{\operatorname{Forb}\left(C_{h}\right)}(p)$, then $K$ is either $K(0,\lceil h / 2\rceil-1)$, $K(1,\lceil h / 3\rceil-1), K(2,0)$ and $h$ is odd, or $K$ has all black vertices (and white or gray edges).

From this point forward, we only restrict ourselves to $p \in(0,1 / 2)$ and CRGs, $K$, with only black vertices and white or gray edges because of Lemma 19. We can immediately address 4 - and 5-cycles. Corollary 20 [12] has appeared before. Corollary 20 was proven in the proof of Lemma 19.

Corollary 20 (Marchant-Thomason [12]). $\operatorname{ed}_{\mathrm{Forb}\left(C_{4}\right)}(p)=p(1-p)$.

Corollary 21. $e d_{\mathrm{Forb}\left(C_{5}\right)}(p)=\min \left\{\frac{p}{2}, \frac{1-p}{2}\right\}$. 
Proof. Thanks to Lemma 19 , we can restrict to $p \in(0,1 / 2)$ and $p$-core CRGs $K \in$ $\mathcal{K}\left(\operatorname{Forb}\left(C_{5}\right)\right.$ for which the vertices are black. Let $g$ denote $g_{K}(p)$ and suppose $g<$ $\min \{p / 2,(1-p) / 2\}$. Let $v_{1}$ have largest weight in $K$ and $v_{2}$ have largest weight in $N_{G}\left(v_{1}\right)$. Since $K$ has no triangles,

$$
\begin{aligned}
\mathrm{d}_{\mathrm{G}}\left(v_{1}\right)+\mathrm{d}_{\mathrm{G}}\left(v_{2}\right) & \leqslant 1 \\
2 \frac{p-g}{p}+\frac{1-2 p}{p}\left(\mathbf{x}\left(v_{1}\right)+\mathbf{x}\left(v_{2}\right)\right) & \leqslant 1 \\
\frac{1-2 p}{p}\left(\mathbf{x}\left(v_{1}\right)+\mathbf{x}\left(v_{2}\right)\right) & \leqslant \frac{2 g-p}{p} .
\end{aligned}
$$

Since the left-hand side is positive, $2 g-p>0$, a contradiction to the assumption that $g<p / 2$.

See Figure 2 and Figure 3.

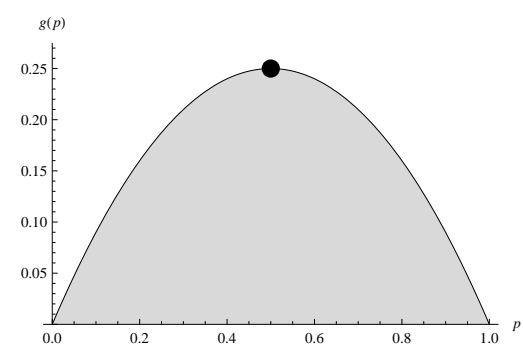

Figure 2: Plot of $e d_{\mathrm{Forb}\left(C_{4}\right)}(p)=$ $p(1-p)$. The boundary of the shaded region is $e d_{\mathrm{Forb}\left(C_{4}\right)}(p)$.

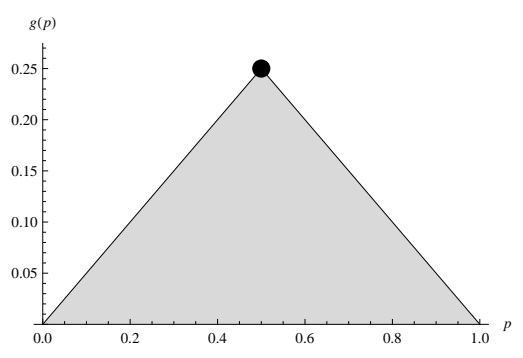

Figure 3: Plot of $e d_{\mathrm{Forb}\left(C_{5}\right)}(p)=$ $\min \{p / 2,(1-p) / 2\}$.

Proposition 22 shows that in order to find CRGs with black vertices, white or gray edges with no $C_{h}$, there are many lengths of gray cycles that are forbidden in the CRG.

Proposition 22. Let $p \in(0,1 / 2)$ and $K$ be a p-core $C R G$ such that $K$ has black vertices and white and gray edges. If $C_{h} \not \rightarrow H$ then $K$ has no gray cycle with length in $\{\lceil h / 2\rceil, \ldots, h\}$

Proof. If $C_{h} \mapsto K$, then each vertex of $K$ receives either one or two vertices that are consecutive on the cycle. Thus, the cycle $K$ must contain is one that corresponds to the contraction of edges of $C_{h}$ that map to a single black vertex of $K$. Since these edges form a matching, the cycle required to be in $K$ has length at least $\lceil h / 2\rceil$ and at most $h$.

In order to deal with $\operatorname{Forb}\left(C_{h}\right)$ for $h \geqslant 6$, we use Proposition 22 along with two major lemmas. Lemma 23 is a general structural lemma and the results on $\operatorname{Forb}\left(C_{h}\right)$ that we give are immediate corollaries. It should be noted that if we write that a CRG, say, "has no gray 4-cycle," we mean so in the subgraph sense, so it does not contain a gray $K_{4}$ either. 
Lemma 23. Let $p \in(0,1 / 2)$ and $K$ be a p-core with black vertices and white or gray edges.

i. If $K$ has no gray edge, then $g_{K}(p)>p$.

ii. If $K$ has neither a gray 3 -cycle nor a gray 4 -cycle, then $g_{K}(p)>p(1-p)$.

iii. If $K$ has no gray 3 -cycle, then $g_{K}(p)>p / 2$.

iv. If $K$ has a gray 3-cycle, but no gray $C_{4}^{+}$(that is, four vertices that induce 5 gray edges $)$, then $g_{K}(p) \geqslant \min \{2 p / 3,(1-p) / 3\}$.

$v$. If $K$ has no gray 4-cycle, then $g_{K}(p)>p(1-p)$ for $p \in(0,1 / 3)$.

vi. If $K$ has a gray $C_{4}^{+}$but no gray $C_{5}^{++}$(that is, five vertices that induce some 5-cycle with two chords), then $g_{K}(p)>\min \{2 p / 3, p(1-p) /(1+p)\}$.

vii. If $K$ has a gray chordless 4 -cycle, but no gray $K_{3,3}^{-}$(that is, a $K_{3,3}$ missing an edge), then $g_{K}(p)>\min \{2 p / 3,2 p(1-p) /(2+p)\}$. Note that $K_{3,3}^{-}$has a 6 -cycle as a subgraph.

The proof of Lemma 23 is in Section 6.1.

Corollary 24. The following are edit distance functions for small cycles:

(6) $e d_{\mathrm{Forb}\left(C_{6}\right)}(p)=\min \left\{p(1-p), \frac{1-p}{2}\right\}$,

(7) $e d_{\mathrm{Forb}\left(C_{7}\right)}(p)=\min \left\{\frac{p}{2}, \frac{p(1-p)}{1+p}, \frac{1-p}{3}\right\}$,

(8) $e d_{\mathrm{Forb}\left(C_{8}\right)}(p)=\min \left\{\frac{p(1-p)}{1+p}, \frac{1-p}{3}\right\}$,

(9) $e d_{\mathrm{Forb}\left(C_{9}\right)}(p)=\min \left\{\frac{p}{2}, \frac{1-p}{4}\right\}$,

(10) $e d_{\operatorname{Forb}\left(C_{10}\right)}(p)=\min \left\{\frac{p(1-p)}{1+2 p}, \frac{1-p}{4}\right\}$, if $p \in[1 / 7,1]$.

See Figures 4-8 for plots of the edit distance functions described in Corollary 24.

\subsection{Proof of Lemma 23}

For ease of notation, in calculations, we sometimes let $g$ denote $g_{K}(p)$.

(i) If $K$ has no gray edges, then for any $v \in V(K), g=p+(1-2 p) \mathbf{x}(v)>p$.

(ii) Let $v_{0} \in V(K)$ have the largest weight and $N_{G}\left(v_{0}\right)=\left\{x_{1}, \ldots, x_{\ell}\right\}$, the gray neighborhood of $v_{0}$. Let $x_{i}=\mathbf{x}\left(v_{i}\right)$ for $i=0,1, \ldots, \ell$. Since there are no gray triangles, there are no gray edges in $N_{G}\left(v_{0}\right)$ and since there are no gray quadrangles, $N_{G}\left(v_{i}\right)-\left\{v_{0}\right\}$ and $N_{G}\left(v_{j}\right)-\left\{v_{0}\right\}$ are disjoint for all distinct $i, j \in\{1, \ldots, \ell\}$. So, 


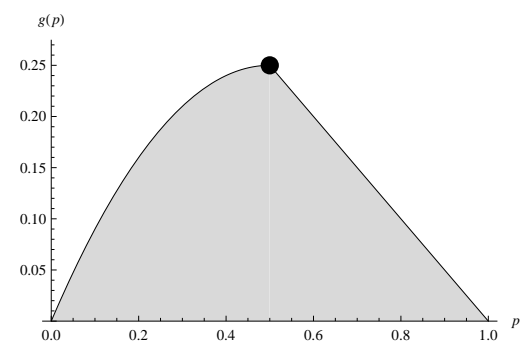

Figure 4: Plot of $e d_{\mathrm{Forb}\left(C_{6}\right)}(p)=$ $\min \{p(1-p),(1-p) / 2\}$.

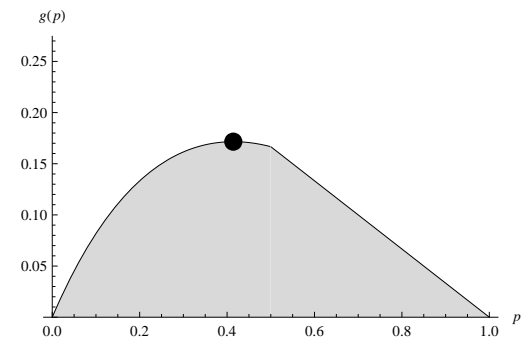

Figure 6: Plot of $e d_{\text {Forb }\left(C_{8}\right)}(p)=$ $\min \{p(1-p) /(1+p),(1-p) / 3\}$.

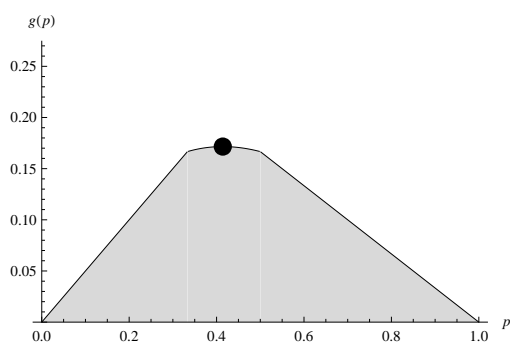

Figure 5: Plot of $e d_{\mathrm{Forb}\left(C_{7}\right)}(p)=$ $\min \{p / 2, p(1-p) /(1+p),(1-$ $p) / 3\}$.

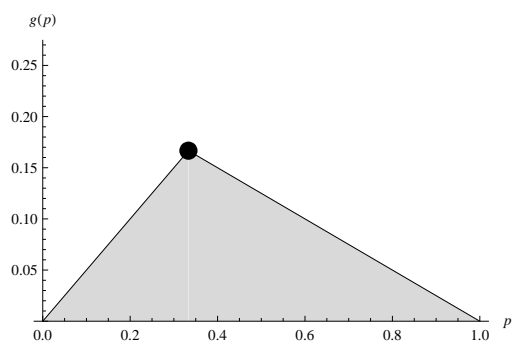

Figure 7: Plot of $e d_{\text {Forb }\left(C_{9}\right)}(p)=$ $\min \{p / 2,(1-p) / 4\}$.

$\left\{v_{0}\right\}, N_{G}\left(v_{0}\right)$ and each $N_{G}\left(v_{i}\right)-\left\{v_{0}\right\}, i=1, \ldots, \ell$ form a family of $\ell+2$ pairwise disjoint sets.

$$
\begin{aligned}
& x_{0}+\mathrm{d}_{\mathrm{G}}\left(v_{0}\right)+\sum_{i=1}^{\ell}\left[\mathrm{d}_{\mathrm{G}}\left(v_{i}\right)-x_{0}\right] \leqslant 1 \\
& x_{0}+\mathrm{d}_{\mathrm{G}}\left(v_{0}\right)+\sum_{i=1}^{\ell}\left[\frac{p-g}{p}+\frac{1-2 p}{p} x_{i}-x_{0}\right] \leqslant 1 \\
& x_{0}+\mathrm{d}_{\mathrm{G}}\left(v_{0}\right)+\ell\left[\frac{p-g}{p}-x_{0}\right]+\frac{1-2 p}{p} \mathrm{~d}_{\mathrm{G}}\left(v_{0}\right) \leqslant 1 \\
& x_{0}+\frac{1-p}{p} \mathrm{~d}_{\mathrm{G}}\left(v_{0}\right)+\ell\left[\frac{p-g}{p}-x_{0}\right] \leqslant 1 .
\end{aligned}
$$

Since $x_{0}$ is the largest weight, $\ell \geqslant \mathrm{d}_{\mathrm{G}}\left(v_{0}\right) / x_{0}$ and as long as $g \geqslant p(1-p)$, we have $\frac{p-g}{p}-x_{0} \geqslant \frac{p-g}{p}-\frac{g}{1-p} \geqslant 0$ by Lemma 16(i). Consequently,

$$
\begin{aligned}
x_{0}+\frac{1-p}{p} \mathrm{~d}_{\mathrm{G}}\left(v_{0}\right)+\frac{\mathrm{d}_{\mathrm{G}}\left(v_{0}\right)}{x_{0}}\left[\frac{p-g}{p}-x_{0}\right] & \leqslant 1 \\
x_{0}^{2}+\mathrm{d}_{\mathrm{G}}\left(v_{0}\right)\left[\frac{p-g}{p}+\frac{1-2 p}{p} x_{0}\right] & \leqslant x_{0}
\end{aligned}
$$




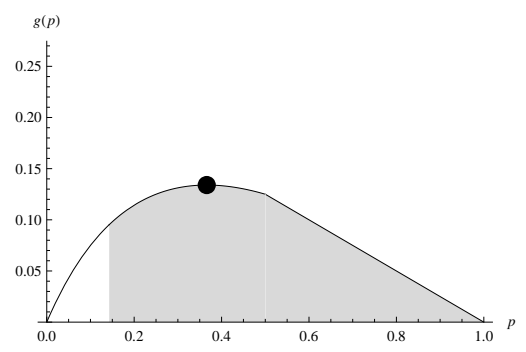

Figure 8: Plot of $e d_{\mathrm{Forb}\left(C_{10}\right)}(p)=\min \{p(1-p) /(1+2 p),(1-p) / 4\}$. An upper bound for $p<1 / 7$ is also on the graph.

$$
\begin{array}{r}
x_{0}^{2}+\left[\frac{p-g}{p}+\frac{1-2 p}{p} x_{0}\right]^{2} \leqslant x_{0} \\
\left(\frac{p-g}{p}\right)^{2}+\left[2 \cdot \frac{p-g}{p} \cdot \frac{1-2 p}{p}-1\right] x_{0}+\left[1+\left(\frac{1-2 p}{p}\right)^{2}\right] x_{0}^{2} \leqslant 0 .
\end{array}
$$

A quadratic expression of the form $c+b x+a x^{2}$ with $a>0$ has a minimum value of $c-b^{2} /(4 a)$.

$$
\begin{gathered}
\left(\frac{p-g}{p}\right)^{2}-\frac{\left(2 \cdot \frac{p-g}{p} \cdot \frac{1-2 p}{p}-1\right)^{2}}{4\left(1+\left(\frac{1-2 p}{p}\right)^{2}\right)} \leqslant 0 \\
4\left(\frac{p-g}{p}\right)^{2}+4\left(\frac{p-g}{p}\right)\left(\frac{1-2 p}{p}\right)-1 \leqslant 0 .
\end{gathered}
$$

So,

$$
\begin{aligned}
\frac{p-g}{p} & \leqslant \frac{1}{2}\left(-\frac{1-2 p}{p}+\sqrt{\left(\frac{1-2 p}{p}\right)^{2}+1}\right) \\
g & \geqslant \frac{1}{2}\left(1-\sqrt{1-4 p+5 p^{2}}\right) .
\end{aligned}
$$

This expression is greater than $p(1-p)$ for all $p \in(0,1 / 2)$.

(iii) By (i), we may assume that $K$ has a gray edge, otherwise $g_{K}(p)>p$. Let $v_{1} v_{2}$ be a gray edge and $x_{i}=\mathbf{x}\left(v_{i}\right)$ for $i=1,2$. Since they have no common gray neighbor,

$$
\begin{aligned}
\mathrm{d}_{\mathrm{G}}\left(v_{1}\right)+\mathrm{d}_{\mathrm{G}}\left(v_{2}\right) & \leqslant 1 \\
2\left(\frac{p-g}{p}\right)+\frac{1-2 p}{p}\left(x_{1}+x_{2}\right) & \leqslant 1
\end{aligned}
$$

Since $x_{1}+x_{2}>0$, we have $g>p / 2$. 
(iv) Let $\left\{v_{1}, v_{2}, v_{3}\right\}$ be a gray triangle in $K$ where $x_{i}=\mathbf{x}\left(v_{i}\right)$ for $i=1,2,3$. Because no pairs of $v_{i}$ can have a common neighbor other than the remaining $v_{j}$,

$$
\begin{aligned}
\sum_{i=1}^{3}\left[\mathrm{~d}_{\mathrm{G}}\left(v_{i}\right)-\left(x_{1}+x_{2}+x_{3}-x_{i}\right)\right]+\left(x_{1}+x_{2}+x_{3}\right) & \leqslant 1 \\
\sum_{i=1}^{3} \mathrm{~d}_{\mathrm{G}}\left(v_{i}\right)-\left(x_{1}+x_{2}+x_{3}\right) & \leqslant 1 \\
3\left(\frac{p-g}{p}\right)+\frac{1-3 p}{p}\left(x_{1}+x_{2}+x_{3}\right) & \leqslant 1 \\
\frac{2 p}{3}+\frac{1-3 p}{3}\left(x_{1}+x_{2}+x_{3}\right) & \leqslant g .
\end{aligned}
$$

If $p<1 / 3$, then $g>2 p / 3$. If $p>1 / 3$, then $x_{1}+x_{2}+x_{3} \leqslant 1$ implies that $g \geqslant(1-p) / 3$.

(v) Let $v_{0} \in V(K)$ have the largest weight. Since there are no gray quadrangles, no member of $N_{G}\left(v_{0}\right)$ has more than one gray neighbor in $N_{G}\left(v_{0}\right)$. Let $N_{G}\left(v_{0}\right)=$ $\left\{x_{1}, x_{1}^{\prime}, \ldots, x_{m}, x_{m}^{\prime}\right\} \cup\left\{x_{2 m+1}, \ldots, x_{\ell}\right\}$, the gray neighborhood of $v_{0}$ such that for $i=1, \ldots, m, x_{i} x_{i}^{\prime}$ is a gray edge. Let $x_{i}=\mathbf{x}\left(v_{i}\right)$ for $i=0,1, \ldots, \ell$. Since there are no gray quadrangles, the gray neighborhoods outside of $\left\{v_{0}\right\} \cup N_{G}\left(v_{0}\right)$ of distinct vertices in $N_{G}\left(v_{0}\right)$ are distinct. Hence,

$$
\begin{aligned}
x_{0}+\mathrm{d}_{\mathrm{G}}\left(v_{0}\right)+\sum_{i=1}^{m}\left[\mathrm{~d}_{\mathrm{G}}\left(v_{i}\right)+\mathrm{d}_{\mathrm{G}}\left(v_{i}^{\prime}\right)-x_{i}-x_{i}^{\prime}-2 x_{0}\right] & \\
+\sum_{j=2 m+1}^{\ell}\left[\mathrm{d}_{\mathrm{G}}\left(v_{j}\right)-x_{0}\right] & \leqslant 1 \\
x_{0}+\mathrm{d}_{\mathrm{G}}\left(v_{0}\right)+\ell\left[\frac{p-g}{p}-x_{0}\right]+\sum_{i=1}^{m}\left(\frac{1-3 p}{p}\right)\left(x_{i}+x_{i}^{\prime}\right) & +\sum_{j=2 m+1}^{\ell}\left(\frac{1-2 p}{p}\right) x_{j} \leqslant 1 \\
\ell\left[\frac{p-g}{p}-x_{0}\right]+x_{0}+\mathrm{d}_{\mathrm{G}}\left(v_{0}\right)+\left(\frac{1-3 p}{p}\right) \mathrm{d}_{\mathrm{G}}\left(v_{0}\right) & \leqslant 1 .
\end{aligned}
$$

Again, we use the fact that $\ell \geqslant \mathrm{d}_{\mathrm{G}}\left(v_{0}\right) / x_{0}$ and $\frac{p-g}{p}-x_{0} \geqslant 0$.

$$
\begin{aligned}
& \frac{\mathrm{d}_{\mathrm{G}}\left(v_{0}\right)}{x_{0}}\left[\frac{p-g}{p}-x_{0}\right]+x_{0}+\left(\frac{1-2 p}{p}\right) \mathrm{d}_{\mathrm{G}}\left(v_{0}\right) \leqslant 1 \\
&\left(\frac{p-g}{p}\right)^{2}+\left[\frac{p-g}{p} \cdot \frac{2-5 p}{p}-1\right] x_{0}+\left[\frac{1-2 p}{p} \cdot \frac{1-3 p}{p}+1\right] x_{0}^{2} \leqslant 0 .
\end{aligned}
$$


Optimizing over $x_{0}$,

$$
\begin{gathered}
\left(\frac{p-g}{p}\right)^{2}-\frac{\left(\left(\frac{p-g}{p}\right)\left(\frac{2-5 p}{p}\right)-1\right)^{2}}{4\left(\left(\frac{1-2 p}{p}\right)\left(\frac{1-3 p}{p}\right)+1\right)} \leqslant 0 \\
\left(\frac{p-g}{p}\right)^{2}\left[4 \frac{1-2 p}{p} \cdot \frac{1-3 p}{p}+4-\left(\frac{2-5 p}{p}\right)^{2}\right] \\
+2 \cdot \frac{p-g}{p} \cdot \frac{2-5 p}{p}-1 \leqslant 0 \\
3\left(\frac{p-g}{p}\right)^{2}+2\left(\frac{2-5 p}{p}\right)\left(\frac{p-g}{p}\right)-1 \geqslant 0
\end{gathered}
$$

So,

$$
\begin{aligned}
\frac{p-g}{p} & \leqslant \frac{1}{3}\left(-\frac{2-5 p}{p}+\sqrt{\left(\frac{2-5 p}{p}\right)^{2}+3}\right) \\
g & \geqslant \frac{2}{3}\left((1-p)-\sqrt{1-5 p+7 p^{2}}\right) .
\end{aligned}
$$

Some calculations show that $g>p(1-p)$ for $p \in(0,1 / 3)$.

(vi) Let the gray $C_{4}^{+}$be denoted $\left\{v_{1}, v_{2}, v_{3}, v_{4}\right\}$ such that all edges are gray except, perhaps $v_{1} v_{3}$. Let $x_{i}=\mathbf{x}\left(v_{i}\right)$ for $i=1,2,3,4$. Without loss of generality, let $x_{2} \geqslant x_{4}$.

No pair $\left(v_{i}, v_{j}\right)$ can have a common gray neighbor except, perhaps $\left(v_{2}, v_{4}\right)$. Denoting $N_{G}(v)$ to be the set of gray neighbors of vertex $v$, the sets $N_{G}\left(v_{1}\right)-\left\{v_{2}, v_{4}\right\}, N_{G}\left(v_{3}\right)-$ $\left\{v_{2}, v_{4}\right\}$ and $N_{G}\left(v_{2}\right)-\left\{v_{1}, v_{3}, v_{4}\right\}$ must be disjoint. So,

$$
\begin{aligned}
\left(\mathrm{d}_{\mathrm{G}}\left(v_{1}\right)-x_{2}-x_{4}\right)+\left(\mathrm{d}_{\mathrm{G}}\left(v_{3}\right)-x_{2}-x_{4}\right) & \\
+\left(\mathrm{d}_{\mathrm{G}}\left(v_{2}\right)-x_{1}-x_{3}-x_{4}\right)+\left(x_{1}+x_{2}+x_{3}+x_{4}\right) & \leqslant 1 \\
3 \cdot \frac{p-g}{p}+\frac{1-2 p}{p}\left(x_{1}+x_{3}\right)+\frac{1-3 p}{p} x_{2}-2 x_{4} & \leqslant 1 \\
2+\frac{1-2 p}{p}\left(x_{1}+x_{3}\right)+\frac{1-3 p}{p} x_{2}-2 x_{4} & \leqslant \frac{3 g}{p} .
\end{aligned}
$$

Solving for $g$,

$$
\begin{aligned}
g & \geqslant \frac{2 p}{3}+\frac{1-2 p}{3}\left(x_{1}+x_{3}\right)+\frac{1-3 p}{3} x_{2}-\frac{2 p}{3} x_{4} \\
& \geqslant \frac{2 p}{3}+\frac{1-2 p}{3}\left(x_{1}+x_{3}\right)+\frac{1-5 p}{3} x_{2} .
\end{aligned}
$$


If $p \leqslant 1 / 5$, then $g>2 p / 3$. If $p>1 / 5$, then we use Lemma $16(\mathrm{i})$, which gives that $x_{2} \leqslant g /(1-p)$. So,

$$
\begin{aligned}
g & \geqslant \frac{2 p}{3}+\frac{1-2 p}{3}\left(x_{1}+x_{4}\right)+\frac{1-5 p}{3}\left(\frac{g}{1-p}\right) \\
& \geqslant \frac{p(1-p)}{1+p}+\frac{(1-2 p)(1-p)}{2(1+p)}\left(x_{1}+x_{4}\right) .
\end{aligned}
$$

Consequently, $g>p(1-p) /(1+p)$.

(vii) Let the gray 4 -cycle be denoted $\left\{v_{1}, v_{2}, v_{3}, v_{4}\right\}$ such that all edges are gray except $v_{1} v_{3}$ and $v_{2} v_{4}$. Let $x_{i}=\mathbf{x}\left(v_{i}\right)$ for $i=1,2,3,4$. If both pairs $\left(v_{1}, v_{3}\right)$ and $\left(v_{2}, v_{4}\right)$ have common neighbors outside of $\left\{v_{1}, v_{2}, v_{3}, v_{4}\right\}$, then a $K_{3,3}$ is formed. So, suppose $v_{2}$ and $v_{4}$ have no common neighbors other than $v_{1}$ and $v_{3}$. Without loss of generality, let $x_{2} \geqslant x_{4}$.

The sets $N_{G}\left(v_{1}\right)-\left\{v_{2}, v_{4}\right\}, N_{G}\left(v_{3}\right)-\left\{v_{2}, v_{4}\right\}$ and $N_{G}\left(v_{2}\right)-\left\{v_{1}, v_{3}\right\}$ must be disjoint. So,

$$
\begin{aligned}
\left(\mathrm{d}_{\mathrm{G}}\left(v_{1}\right)-x_{2}-x_{4}\right)+\left(\mathrm{d}_{\mathrm{G}}\left(v_{3}\right)-x_{2}-x_{4}\right) & \\
+\left(\mathrm{d}_{\mathrm{G}}\left(v_{2}\right)-x_{1}-x_{3}\right)+\left(x_{1}+x_{2}+x_{3}+x_{4}\right) & \leqslant 1 \\
3 \frac{p-g}{p}+\frac{1-2 p}{p}\left(x_{1}+x_{3}\right)+\frac{1-3 p}{p} x_{2}-x_{4} & \leqslant 1 \\
2+\frac{1-2 p}{p}\left(x_{1}+x_{3}\right)+\frac{1-3 p}{p} x_{2}-x_{4} & \leqslant \frac{3 g}{p} .
\end{aligned}
$$

Solving for $g$,

$$
\begin{aligned}
g & \geqslant \frac{2 p}{3}+\frac{1-2 p}{3}\left(x_{1}+x_{3}\right)+\frac{1-3 p}{3} x_{2}-\frac{p}{3} x_{4} \\
& \geqslant \frac{2 p}{3}+\frac{1-2 p}{3}\left(x_{1}+x_{3}\right)+\frac{1-4 p}{3} x_{2} .
\end{aligned}
$$

If $p \leqslant 1 / 4$, then $g>2 p / 3$. If $p>1 / 4$, then we use Lemma 16(i), which gives that $x_{2} \leqslant g /(1-p)$.

$$
\begin{aligned}
g & \geqslant \frac{2 p}{3}+\frac{1-2 p}{3}\left(x_{1}+x_{4}\right)+\frac{1-4 p}{3}\left(\frac{g}{1-p}\right) \\
& \geqslant \frac{2 p(1-p)}{2+p}+\frac{(1-2 p)(1-p)}{2+p}\left(x_{1}+x_{4}\right) .
\end{aligned}
$$

Consequently, $g>2 p(1-p) /(2+p)$.

This concludes the proof of Lemma 23. 


\subsection{Proof of Corollary 24}

(6) $C_{6}$ : Lemma 19 gives that the function stated above is $\gamma_{\operatorname{Forb}\left(C_{6}\right)}(p)$ and so $e d_{\text {Forb }\left(C_{6}\right)}(p) \leqslant \min \left\{p(1-p), \frac{1-p}{2}\right\}$. By Lemma 19, we only need to consider $p \in(0,1 / 2)$ and $K$ being a black-vertex $p$-core $\mathrm{CRG}$ in $\mathcal{K}\left(\operatorname{Forb}\left(C_{6}\right)\right)$ for which $g_{K}(p)<\gamma_{\text {Forb }\left(C_{6}\right)}(p)$. By Proposition 22, $K$ has neither a 3 -cycle nor a 4 -cycle. Lemma 23(ii) gives that $g_{K}(p) \geqslant p(1-p)$. So, there is no such $K$ and the corollary follows.

(7) $C_{7}$ : The function stated above is $\gamma_{\operatorname{Forb}\left(C_{7}\right)}(p)$. Let $p \in(0,1 / 2)$ and suppose $K$ is a black-vertex $p$-core $\mathrm{CRG}$ in $\mathcal{K}\left(\operatorname{Forb}\left(C_{7}\right)\right)$ for which $g_{K}(p)<\gamma_{\mathrm{Forb}\left(C_{7}\right)}(p)$. By Proposition 22, $K$ has no gray 4-cycle.

Since $K$ has no gray 4-cycle, then by Lemma 23(ii), either $g_{K}(p)>p(1-p)$ or $K$ has a gray 3 -cycle. In terms of the former, it is trivial that this is a contradiction to $g_{K}(p)<\gamma_{\mathrm{Forb}\left(C_{7}\right)}(p)$ for $p \in(0,1 / 2)$, so we assume that $G$ has a gray 3 -cycle.

If $K$ has a gray 3 -cycle but no $C_{4}^{+}$, then by Lemma 23(iv), we have $g_{K}(p)>$ $\min \{2 p / 3,(1-p) / 3\}$. Straightforward calculations verify that this is a contradiction to $g_{K}(p)<\gamma_{\operatorname{Forb}\left(C_{7}\right)}(p)$ for $p \in(0,1 / 2)$.

(8) $C_{8}$ : The proof is the same as for Corollary 7.

(9) $C_{9}$ : The function stated above is $\gamma_{\mathrm{Forb}\left(C_{9}\right)}(p)$. Let $p \in(0,1 / 2)$ and suppose $K$ is a black-vertex $p$-core $\mathrm{CRG}$ in $\mathcal{K}\left(\operatorname{Forb}\left(C_{9}\right)\right)$ for which $g_{K}(p)<\gamma_{\operatorname{Forb}\left(C_{9}\right)}(p)$. By Proposition 22, $K$ has no gray $C_{5}^{++}$.

Since $K$ has no gray $C_{5}^{++}$, then by Lemma $23\left(\right.$ vi), either $g_{K}(p)>\min \{2 p / 3, p(1-$ $p) /(1+p)\}$ or $K$ has no gray $C_{4}^{+}$. In terms of the former, straightforward calculations verify that this is a contradiction to $g_{K}(p)<\gamma_{\mathrm{Forb}\left(C_{9}\right)}(p)$ for $p \in(0,1 / 2)$, so we assume that $G$ has no gray $C_{4}^{+}$.

If $K$ has no gray $C_{4}^{+}$, then by Lemma $23(\mathrm{iv})$, either $g_{K}(p)>\min \{2 p / 3,(1-p) / 3\}$ or $K$ has no gray 3 -cycle. In terms of the former, it is trivial that this is a contradiction to $g_{K}(p)<\gamma_{\operatorname{Forb}\left(C_{9}\right)}(p)$ for $p \in(0,1 / 2)$, so we assume that $G$ has no gray 3 -cycle. If that is the case, however, Lemma 23(iii) gives that $g_{K}(p)>p / 2$, a contradiction. So, there is no such $K$ for which $g_{K}(p)<\gamma_{\operatorname{Forb}\left(C_{9}\right)}(p)$ and the corollary follows.

(10) $C_{10}$ : The function stated above is $\gamma_{\operatorname{Forb}\left(C_{10}\right)}(p)$. Let $p \in(0,1 / 2)$ and suppose $K$ is a black-vertex $p$-core $\mathrm{CRG}$ in $\mathcal{K}\left(\operatorname{Forb}\left(C_{10}\right)\right)$ for which $g_{K}(p)<\gamma_{\mathrm{Forb}\left(C_{9}\right)}(p)$. By Proposition 22, $K$ has no gray $C_{5}^{++}$.

Since $K$ has no gray $C_{5}^{++}$, then by Lemma $23\left(\right.$ vi), either $g_{K}(p)>\min \{2 p / 3, p(1-$ $p) /(1+p)\}$ or $K$ has no gray $C_{4}^{+}$. In terms of the former, straightforward calculations verify that this is a contradiction to $g_{K}(p)<\gamma_{\operatorname{Forb}\left(C_{10}\right)}(p)$ for $p \in[1 / 7,1 / 2)$, so we assume that $K$ has no gray $C_{4}^{+}$. 
If $K$ has no gray $C_{4}^{+}$, then by Lemma $23(\mathrm{iv})$, either $g_{K}(p)>\min \{2 p / 3,(1-p) / 3\}$ or $K$ has no gray 3 -cycle. In terms of the former, it is trivial that this is a contradiction to $g_{K}(p)<\gamma_{\mathrm{Forb}\left(C_{10}\right)}(p)$ for $p \in[1 / 7,1 / 2)$, so we assume that $K$ has no gray 3 -cycle. If $K$ has no gray 3-cycle, then by Lemma 23(ii), either $g_{K}(p)>p(1-p)$ or $K$ has a gray 4-cycle. In terms of the former, it is trivial that this is a contradiction to $g_{K}(p)<\gamma_{\mathrm{Forb}\left(C_{10}\right)}(p)$ for $p \in(0,1 / 2)$, so we assume $K$ has a 4 -cycle, but since it cannot be $C_{4}^{+}$, it must be a gray chordless 4 -cycle.

If $K$ has a chordless gray 4-cycle, then by Lemma 23(vii), either $g_{K}(p)>$ $\min \{2 p / 3,2 p(1-p) /(2+p)\}$ or $K$ has a gray $K_{3,3}^{-}$. In terms of the former, straightforward calculations verify that this is a contradiction to $g_{K}(p)<\gamma_{\operatorname{Forb}\left(C_{10}\right)}(p)$ for $p \in[1 / 7,1 / 2)$, so we assume that $K$ has a gray $K_{3,3}^{-}$. However, as observed in Lemma 23, this contains a gray 6 -cycle, which is a contradiction to $K \in \mathcal{K}\left(\operatorname{Forb}\left(C_{10}\right)\right)$.

This concludes the proof of Corollary 24 .

\section{Conclusions}

We provide a conjecture with some interesting implications. Recall that $G(n, p)$ denotes the Erdős-Rényi random graph on $n$ vertices with edge-probability $p$. The hereditary property $\mathcal{H}=\operatorname{Forb}\left(G\left(n_{0}, p_{0}\right)\right)$ is a random variable.

Conjecture 25. Fix $p_{0} \in(0,1)$ and let $\mathcal{H}=\operatorname{Forb}\left(G\left(n_{0}, p_{0}\right)\right)$. Then

$$
e d_{\mathcal{H}}(p)=(1+o(1)) \frac{2 \log _{2} n_{0}}{n_{0}} \min \left\{\frac{p}{-\log _{2}\left(1-p_{0}\right)}, \frac{1-p}{-\log _{2} p_{0}}\right\}
$$

with probability approaching 1 as $n_{0} \rightarrow \infty$.

The functions that define this bound are of the form $p /(\chi-1)$ and $(1-p) /(\bar{\chi}-1)$.

Conjecture 25 was proved for the case $p_{0}=1 / 2$ by Alon and Stav [3]. If it is true in general, then it implies that $p_{\mathcal{H}}^{*}=\frac{\log \left(1-p_{0}\right)}{\log p_{0}\left(1-p_{0}\right)}$, which is only equal to $p_{0}$ itself when $p_{0} \in\{0,1 / 2,1\}$. Recall that $e d_{\mathcal{H}}(p)=\lim _{n \rightarrow \infty} \operatorname{dist}(G(n, p), \mathcal{H}) /\left(\begin{array}{c}n \\ 2\end{array}\right)$ and it achieves its maximum at $p_{\mathcal{H}}^{*}$. Informally, the conjecture implies that it is harder to edit away copies of $G\left(n_{0}, p_{0}\right)$ from $G\left(n, p_{\mathcal{H}}^{*}\right)$ than it is from $G\left(n, p_{0}\right)$. This seems to be rather counterintuitive.

If Conjecture 25 is false, then it implies that there is more information about the structure of random graphs than is revealed by just the chromatic numbers.

\section{Acknowledgements}

I would like to thank Maria Axenovich and József Balogh for conversations which have improved the results. I would like to thank Andrew Thomason for some useful conversations and for directing me to [12]. I would also like to thank Tracy McKay for valuable discussions which deepened my understanding of previous results. 
Thank you to Ed Marchant for finding an error in a previous version of this manuscript. Figures are made by Mathematica and WinFIGQT.

\section{References}

[1] V. E. Alekseev. On the entropy values of hereditary properties. Discrete Math. Appl. 3: 191-199, 1993.

[2] N. Alon and A. Stav. What is the furthest graph from a hereditary property? Random Structures Algorithms 33(1): 87-104, 2008.

[3] N. Alon and A. Stav. The maximum edit distance from hereditary graph properties. J. Combin. Th. Ser. B 98(4): 672-697, 2008.

[4] N. Alon and A. Stav. Stability type results for hereditary properties. J. Graph Theory 62(1): 65-83, 2009.

[5] N. Alon and A. Stav. Hardness of edge-modification problems. Theoret. Comput. Sci. 410(47-49): 4920-4927, 2009.

[6] M. Axenovich, A. Kézdy and R. Martin. On the editing distance of graphs. J. Graph Theory 58(2): 123-138, 2008.

[7] M. Axenovich and R. Martin. Avoiding patterns in matrices via a small number of changes. SIAM J. Discrete Math. 20(1): 49-54, 2006.

[8] J. Balogh and R. Martin. Edit distance and its computation. Electron. J. Combin. 15(1): P20, 2008.

[9] B. Bollobás and A. Thomason. Hereditary and monotone properties of graphs. In The mathematics of Paul Erdös, II, vol. 14 of Algorithm. Combin., pages 70-78, Springer, 1997.

[10] B. Bollobás and A. Thomason. The structure of hereditary properties and colourings of random graphs. Combinatorica 20(2): 173-202, 2000.

[11] W. G. Brown. On graphs that do not contain a Thomsen graph. Canad. Math. Bull. 9: 281-285, 1966.

[12] E. Marchant and A. Thomason. Extremal graphs and multigraphs with two weighted colours. In Fete of combinatorics and computer science, volume 20 of Bolyai Soc. Math. Stud., pages 239-286, János Bolyai Math. Soc., 2010.

[13] O. Pikhurko. An exact Turán result for the generalized triangle. Combinatorica 28(2): 187-208, 2008.

[14] H. J. Prömel and A. Steger. Excluding induced subgraphs: quadrilaterals. Random Structures Algorithms 2: 55-71, 1991.

[15] H. J. Prömel and A. Steger. Excluding induced subgraphs II: extremal graphs. Discrete Appl. Math. 44: 283-294, 1993.

[16] H. J. Prömel and A. Steger. Excluding induced subgraphs III: a general asymptotic. Random Structures Algorithms 3: 19-31, 1992. 
[17] D. C. Richer. Graph Theory and Combinatorial Games. Ph.D. thesis, University of Cambridge, 2000.

[18] A. F. Sidorenko. Boundedness of optimal matrices in extremal multigraph and digraph problems. Combinatorica 13(1): 109-120, 1993.

[19] A. Thomason. Private communication. 